\title{
Pratiques
}

Linguistique, littérature, didactique

$157-158 \mid 2013$

Théories et pratiques des genres

\section{L'approche par genres discursifs dans l'enseignement du français langue étrangère et langue de scolarisation}

Jean-Claude Beacco

\section{OpenEdition}

Journals

Édition électronique

URL : https://journals.openedition.org/pratiques/3838

DOI : 10.4000/pratiques.3838

ISSN : 2425-2042

Éditeur

Centre de recherche sur les médiations (CREM)

\section{Édition imprimée}

Date de publication : 1 juin 2013

Pagination : 189-200

Référence électronique

Jean-Claude Beacco, «L'approche par genres discursifs dans l'enseignement du français langue étrangère et langue de scolarisation », Pratiques [En ligne], 157-158 | 2013, mis en ligne le 18 décembre 2017, consulté le 21 septembre 2021. URL : http://journals.openedition.org/pratiques/3838; DOI : https://doi.org/10.4000/pratiques.3838 


\title{
L'approche par genres discursifs dans l'enseignement du français langue étrangère et langue de scolarisation
}

\author{
Jean-Claude Beacco \\ GRAC-DILTEC, EA 2288. Université Paris III
}

La didactique des langues doit à D. Hymes le concept fondateur de compétence de communication et celui de communauté de communication (« une communauté possédant en commun des règles régissant le déroulement et l'interprétation de la parole, et des règles régissant l'interprétation d'au moins une variété linguistique »(1972 : 133). Mais elle lui doit aussi ${ }^{(1)}$, comme le soulignent Coste, de Pietro et Moore (2012:113), celui de genre de discours, qui n'est pourtant que l'une des 16 composantes (la dernière, d'ailleurs) du modèle SPEAKING, mais qui a été au cœur d'une « opérationalisation didactique de la compétence de communication » en Suisse.

Au-delà du contexte Suisse, nous décrirons la place stratégique du concept de genre de discours en didactique des langues étrangères, en particulier dans celle de l'enseignement du français comme langue étrangère mais aussi en didactique de l'enseignement du français langue première/des langues de scolarisation, comme matière spécifique et surtout comme vecteur de la transmission des connaissances dans les autres matières scolaires (par ex. mathématiques, géographie, sciences de la vie et de la terre...). Ces deux approches de la structuration des enseignements par objectifs définis en termes de genres de discours sont certes distinctes, mais elles relèvent bien d'un principe commun, qu'il nous a déjà été donné d'exposer, pour ce qui concerne le français langue étrangère (par exemple, Beacco, 1991) et, plus récemment, pour ce qui est des langues de scolarisation (par exemple, Beacco, 2009). Nous reviendrons ici sur certaines formes de sa mise en œuvre dans la constitution des programmes d'enseignement.

(1) Même si d'autres origines sont aussi envisageables. 


\section{Genre de discours et répertoire de genres individuel}

Au préalable, il importe de préciser les caractéristiques de ce concept que nous considérons comme définitoires, car celui-ci peut être construit selon différents points de vue épistémologiques, par exemple, au niveau des signifiants, sous l'angle de ses régularités de composition (la dispositio de la rhétorique antique) ou des caractéristiques formelles des textes produits ou encore, au niveau linguistique englobant, en termes d'ancrage social ou de forme de communication (d'après Charaudeau, $2002: 280$ ).

Pour le présent propos, nous retiendrons en premier lieu (d'après Beacco, 2004) que tout locuteur se définit par son répertoire discursif (ou répertoire de genres/générique), c'est-à-dire par les genres de la communication verbale dont il dispose. Ce répertoire discursif peut être réalisé dans plusieurs langues, tant et si bien que répertoire discursif est alors coextensif à répertoire plurilingue (Coste, Moore et Zarate, 2009), comme ensemble des langues différentes connues de chacun et des formes discursives maîtrisées dans ces langues ; ce répertoire est constitué des genres de discours pour lesquels un locuteur a acquis des compétences différentes, à des niveaux de maîtrise eux-mêmes différents.

On pose ensuite que la notion de genre de discours (ou genre discursif) est une représentation métalinguistique des formes de la communication propres à une communauté de communication donnée ${ }^{(2)}$. Les dénominations des genres discursifs constituent les filtres à travers lesquels les locuteurs appréhendent la communication verbale. Ils sont en mesure d'en comprendre et d'en produire certains, mais tous les genres de discours n'entrent pas dans la composition des répertoires individuels. Ceux-ci sont éminemment variables, même si l'on peut considérer qu'une forme minimale commune de répertoire est constituée par la maîtrise de genres oraux interactifs (conversations de service, dispute, conversation à bâtons rompus...) ou non interactifs (anecdote), de certains genres écrits (lettres et messages personnels) et de genres surtout reconnus, car fréquentés dans les médias écrits (faits divers) ou audiovisuels (journal télévisé, interview). «Toute situation quotidienne stable comporte un auditoire organisé d'une certaine façon, et par conséquent un certain répertoire de petits genres quotidiens » (Bakhtine M., 1929, cité par Todorov T., 1981 : 127). Cette expérience générique est largement partagée dans une même communauté de communication, mais celle-ci comporte aussi des communautés restreintes de type professionnel, économique, religieux, sportif, politique, scientifique..., qui ont été décrites au moyen du terme de topologie discursive (Beacco, 1999 : 13-16). Les genres propres à ces communautés « spécialisées » doivent être appris, alors que tous ne sont pas tous enseignés explicitement. On est ainsi amené à considérer que tout enseignement constitue un élargissement du répertoire de genres minimal commun ou une forme d'élargissement de l'expérience générique antérieure.

Enfin, on soulignera que les genres discursif peuvent recevoir des formes linguistiques dont le degré de spécificité est variable. Il convient alors de ne pas

(2) A l'exclusion des genres littéraires à considérer comme savants (épopée, tragédie, sonnet...). 
confondre la variabilité des textes selon leur degré de conformité au genre dont ils relèvent avec la variabilité des genres entre eux :

« les formes des régularités génériques sont elles-mêmes diverses, non dans leurs contenus mais dans les modes dont elles informent chaque genre. Ainsi, certains sont très marqués par les objets de discours qu'ils construisent (par exemple, les faits divers), mais d'autres présentent une gamme ouverte d'objets de discours (conversation entre amis). Les constantes de linéarité (succession régulière et prévisible d'éléments discursifs) sont très évidentes pour certains (constats, rapports de police, articles scientifiques des sciences exactes), mais quasi nulles pour d'autres (articles de divulgation scientifique dans la presse quotidienne). Les uns sont formulaires, donnent prise à l'analyse automatique et permettent de produire de nouveaux textes par substitution lexicale (petites annonces, faire-part, vœux...), d'autres ne sont ni codifiés ni répétitifs mais pourtant apparentés. Les structures syntaxiques des phrases peuvent caractériser certains (écrits techniques, horoscopes, dictionnaires), alors que peu de régularités de cette nature sont décelables dans les homélies ou les journaux intimes. D. Maingueneau (2002 : 322-323) propose une nouvelle classification des genres discursifs précisément à partir du niveau d'intensité des contraintes qui informent leurs réalisations verbales : il distingue, dans l'ordre décroissant de conditionnement du modèle générique sur les textes, les genres formulaires ou à faible variation (construits à partir de formules et de schémas), ceux commandés par des scripts, ceux qui mettent en jeu des scénographies dont le choix revient à l'énonciateur, ceux qui impliquent de la créativité (comme la publicité) et ceux pour lesquels il n'existe pas de format préétablis, mais uniquement des zones génériques déterminées. » (Beacco 2004 : 115)

Maîtriser un nouveau genre discursif (en réception ou en production) signifie être capable de produire (ou de reconnaître) des textes conformes aux caractéristiques de celui-ci (lesquelles sont très probablement distinctes des caractéristiques des genres déjà connus) et de le faire en ayant conscience du degré et des formes de variation admissibles, propres à chaque genre.

On retiendra de ces spécifications que :

- la notion de genre de discours est constitutive de l'expérience communicative de tout locuteur ;

- chaque locuteur dispose d'un répertoire de genres et de langues que l'éducation a pour responsabilité de valoriser et d'élargir ;

— bien des genres ne relèvent pas de l'expérience ordinaire des échanges verbaux mais supposent une forme d'acquisition qui permette de les comprendre et de les produire.

Ainsi apprendre une langue étrangère revient à s'approprier une langue inconnue par de nouveaux genres et accéder aux connaissances à l'École revient à accéder à de nouveaux genres dans une même langue, l' « identité » présumée de cette langue pouvant tendre à masquer la diversité des formes discursives.

\section{L'approche de l'enseignement des langues par genres de discours}

Si l'on replace la question des genres de discours dans le contexte actuel relatif à la réflexion sur les méthodologies d'enseignement des langues, on ne peut 
pas éviter de convoquer le Cadre européen commun de référence pour les langues (Conseil de l'Europe, 2001 ; désormais le Cadre).

\subsection{Les genres de discours et le Cadre}

Cet instrument indique clairement qu'il ne privilégie aucune méthodologie d'enseignement particulière ${ }^{(3)}$ mais la spécification de la compétence à communiquer langagièrement qu'il utilise en compétence relative aux genres de discours est permise par ce texte. Dans le Cadre, on a effectivement préféré le concept de compétence pragmatique et celui de texte à celui de genre de discours, pour caractériser la compétence proprement communicationnelle et langagière de l'utilisateur/apprenant. Mais la compétence pragmatique est donnée dans le Cadre comme se rapportant aussi aux régularités des discours. La notion de genre de textes y est présente, mais elle est toujours associée à celle de type de textes et finalement peu distinguée de celle-ci.

Le Cadre est, par définition un ouvrage consensuel, destiné qu'il est à assurer la transparence chez les spécialistes de l'enseignement des langues Il n'est donc pas étonnant qu'il reprenne une certaine tradition (très présente en didactique du français langue étrangère) qui, dès le début des années 1975, voyait dans la description des régularités linguistiques des genres discursifs l'une des bases possibles pour l'organisation des méthodologies d'enseignement des langues étrangères d'inspiration communicative. Le concept de genre de discours est en effet indispensable en ce qui concerne l'enseignement/apprentissage des langues, car les genres de discours sont des formes de communication verbale propres à des communautés discursives c'est-à-dire, d'une certaine manière et indirectement, à des langues. Certains genres n'ont pas d'équivalent d'une langue à l'autre ou encore ils ne coïncident que partiellement ou superficiellement. Apprendre une langue revient en fait à gérer le dépaysement discursif qui résulte du contact entre deux cultures discursives, celle de la communauté discursive d'origine des apprenants et celle de la langue cible.

\subsection{Des référentiels de programmes par genres de discours}

Certains instruments destinés à servir à la constitution de programmes de langues, regroupés sous la dénomination de Description des niveaux de référence $d u$ CECR par langue (DNR) ${ }^{(4)}$, s'emploient à transposer les descripteurs de compétences du Cadre, indépendants des langues, en formes particulières des langues à apprendre. Pour le français, cette génération de référentiels (intitulés Niveaux pour le français) ${ }^{(5)}$ utilise les catégories de genre de discours et de répertoire de discours, au niveau le plus englobant (chapitre 2), les autres catégorisations sont d'ordre inférieur dans la hiérarchie des spécifications emboîtées qui en constituent l'armature, comme l'illustre le schéma ci-après.

(3) Cadre p. 110.

(4) http://www.coe.int/t/dg4/linguistic/DNR_FR.asp.

(5) Beacco et al. : Niveaux pour le français: A1.1, A1, A2, B1, B2, Paris, Didier. 


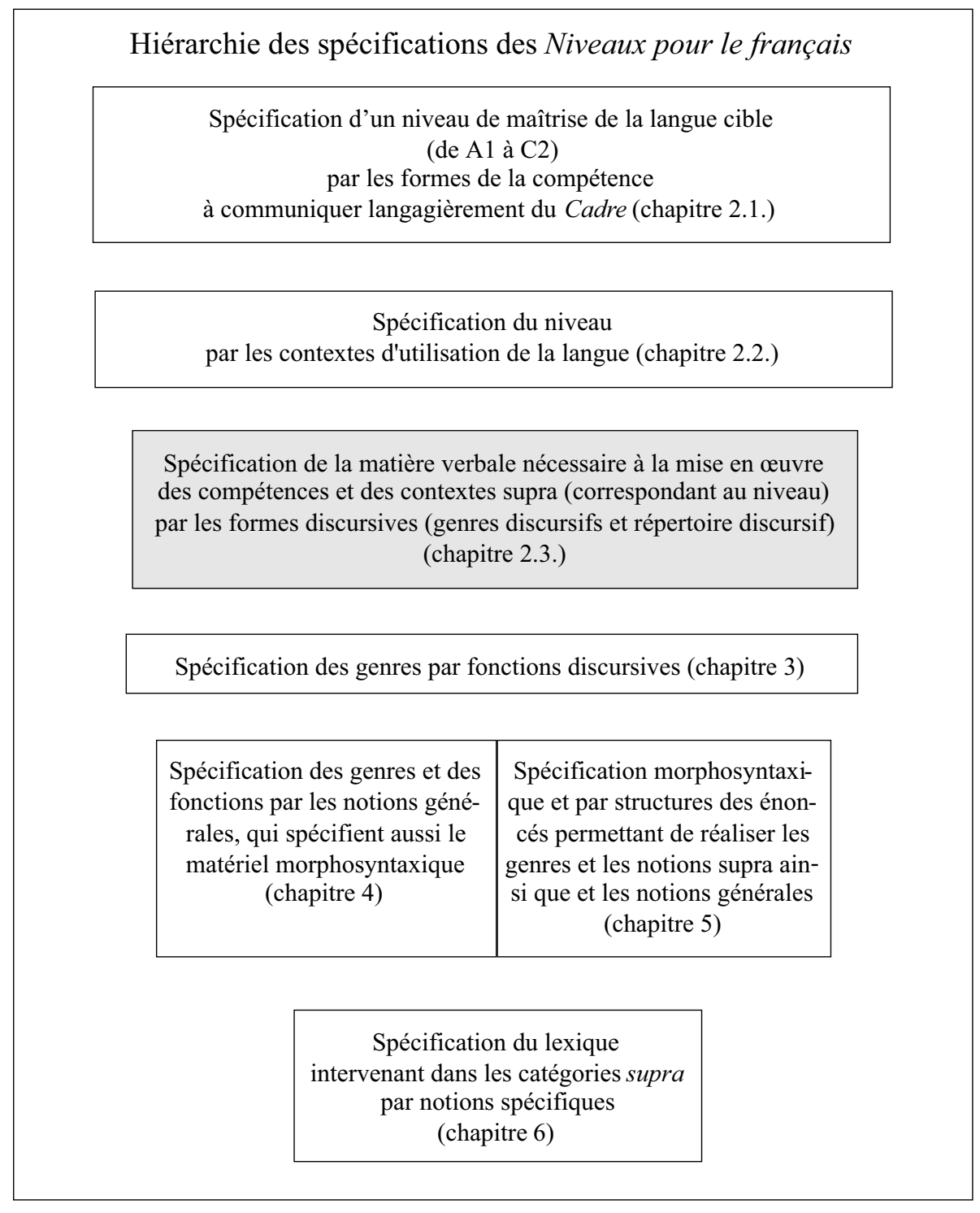

Dans les Niveaux pour le français ${ }^{(6)}$, un niveau de compétence communicationnelle donné en français est ainsi caractérisé, au plus haut niveau linguistique (immédiatement " sous » les descripteurs qualitatifs " universels » - c'est-àdire non propres à une langue — du Cadre) par le répertoire de genres de discours que l'on souhaite voir les apprenants se constituer. On donnera comme exemple le répertoire discursif considéré comme caractéristique du niveau A2 uniquement pour les activités de réception/compréhension écrite :

(6) Mais aussi dans (2008) : Profilo italiano. Descrizione dei livelli di riferimento del Quadro europeo commune per la lingua italiana, Centro valutazione certificazione linguitica, Università per Stranieri di Perugia et dans $(2006,2007)$ : Plan curricular del Instituto Cervantes (A1-A2, B1-B2,C1-C2), Instituto Cervantes, Biblioteca Nueva, Madrid, chapitre 7. 


\begin{tabular}{|c|c|}
\hline \multicolumn{2}{|r|}{ Réception écrite niveau A2 } \\
\hline Domaine privé & $\begin{array}{l}\text { - correspondance familiale, amicale } \\
\text { - mots, billets (avec graphie manuelle interprétable), } \\
\text { messages (dont SMS) }\end{array}$ \\
\hline Écrits publics & $\begin{array}{l}\text { - panneaux indicateurs, signalétique routière et touristique } \\
\text { - plans, noms de rues, de stations } \\
\text { - noms de magasins, d'édifices publics } \\
\text { - plaques commémoratives } \\
\text { - petites annonces (immobilières, location surtout) }\end{array}$ \\
\hline Instructions & $\begin{array}{l}\text { - méthodes (dont méthodes de langue...) } \\
\text { - recettes (cuisine, pâtisserie, cocktails...), règles de jeux } \\
\text { - modes d'emploi simples }\end{array}$ \\
\hline $\begin{array}{l}\text { Textes à valeur légale, } \\
\text { formulaires administratifs, } \\
\text { documents à caractère officiel }\end{array}$ & $\begin{array}{l}\text { - étiquettes, emballages, date de péremption, composition, } \\
\text { vignette de médicament } \\
\text { - bail } \\
\text { - bulletin de salaire } \\
\text { - diplômes, relevés de notes, de résultats } \\
\text { - passeport, permis de conduire, carte de séjour, certificats } \\
\text { (de naissance, d'assurance...) et autres documents } \\
\text { personnels } \\
\text { - textes et formulaires déjà connus dans la culture } \\
\text { d'origine : déclaration de devises, invitation, faire-part... } \\
\text { - billets de banque, timbres, timbres fiscaux } \\
\text { - reçu, addition, ticket de caisse } \\
\text { - titre de transport } \\
\text { - curriculum vitae }\end{array}$ \\
\hline $\begin{array}{l}\text { Presse quotidienne nationale, } \\
\text { presse hebdomadaire } \\
\text { d'information } \\
\text { (textes relatifs à des situations } \\
\text { ou des sujets entrant dans } \\
\text { l'expérience du monde et } \\
\text { faisant partie des } \\
\text { connaissances } \\
\text { encyclopédiques de } \\
\text { l'utilisateur) }\end{array}$ & $\begin{array}{l}\text { - sommaire } \\
\text { - informations nationales et internationales (titre explicites) } \\
\text { - titres intérieurs } \\
\text { - faits divers } \\
\text { - petites annonces (immobilière) } \\
\text { - légendes de cartes, dessins, croquis, tableaux de données } \\
\text { numériques... } \\
\text { - nouvelles brèves, comprises globalement (relevant du } \\
\text { domaine d'expérience des apprenants) } \\
\text { - météo } \\
\text { - programme de télévision } \\
\text { - carnet } \\
\text { - horoscope (surtout ceux des quotidiens, compris } \\
\text { globalement et partiellement) } \\
\text { - conseils, instructions (régime alimentaire, santé recettes } \\
\text { de cuisine...) }\end{array}$ \\
\hline $\begin{array}{l}\text { Autres types de presse : revues } \\
\text { et magazines « féminins », } \\
\text { presse pour adolescents, presse } \\
\text { spécialisée, en général }\end{array}$ & - pour les genres de discours, voir ci-dessus \\
\hline $\begin{array}{l}\text { Textes relatifs à la } \\
\text { transmission de connaissances }\end{array}$ & $\begin{array}{l}\text { - manuels, cours } \\
\text { - dictionnaires, dictionnaires de langue, - grammaires } \\
\text { - articles brefs dans des ouvrages ou publications de } \\
\text { divulgation }\end{array}$ \\
\hline
\end{tabular}

(Beacco et al. 2008 : 46-47) 
Cet inventaire des genres discursifs visés en A2 ne dispense pas de vérifier si un texte donné, relevant d'un genre répertorié présente bien les caractéristiques générales définissant la compétence de type A2. Et cette identification par les genres ne tient pas compte du fait qu'un genre peut être compris ou produit à différents niveaux de maîtrise qu'on ne peut pas toujours relier de façon simple un genre à un niveau. Il n'empêche que placer les genres de discours au degré le plus élevé de structuration d'un programme revient à inviter à construire des séquences d'enseignement centrées sur des textes relevant de ces genres et non sur des thèmes, des contenus de prédilection, des champs lexicaux, des structures syntaxiques ou des catégories morphologiques, des actes de langage... toutes formes d'organisation de l'enseignement où la notion de genre et de texte sont ignorées.

Donner aux genres de discours une fonction organisatrice déterminante pour la spécification des contenus d'enseignement (auxquels sont subordonnées les autres composantes : lexique, morphosyntaxe, contenus...) ne constitue que la forme la plus détaillée de l'approche par compétences (réception orale, écrite, interaction orale...), puisque que tout genre entre dans l'une de ces catégories larges, nommées activités dans le Cadre. On soutiendra volontiers que 1'approche par genre de discours n'est qu'une autre formulation de l'approche communicative de l'enseignement des langues

Cette « approche par genre (de discours) » n'a cependant guère fait parler d'elle en didactique des langues. A. Burns (2013) dans sa notice sur genre et genre based teaching relève que cette stratégie est à relier, dans le domaine anglophone, à l'enseignement sur objectifs spécifiques, ce qui a aussi été le cas en France. Elle se fait l'écho de nombre de critiques qui voient dans cette approche une forme trop prescriptive d'enseignement et qui la considèrent comme un dispositif qui va à l'encontre de la créativité naturelle des apprenants. Ce n'est pas ici le lieu d'entrer dans ce débat mais on peut estimer que celui-ci appelle des réponses diversifiées suivant le degré de structuration (variable) des genres eux-mêmes. Les genres formulaires très contraints (genre à caractère juridique et administratif, par exemple) ne laissent pas de latitude à l'énonciateur et peuvent sans risques faire l'objet d'un enseignement mémoriel assez mécaniste. Tous les autres genres ne sont pas réglés par des conditions de production strictes, mais bien par des faisceaux de caractéristiques attendues qui assurent la propriété d'un texte par rapport au genre dont il relève. Ces régulations souples autorisent du jeu énonciatif, qui constitue un espace pour l'ajustement des textes aux destinataires ou aux stratégies de l'énonciateur. Une telle conception différenciée des genres de discours devrait être en mesure de ne pas rendre les apprentissages purement imitatifs, surtout si les apprenants sont exposés non à des prototypes de textes appropriés mais à un éventail d'échantillons de textes-cible, extraits de la circulation sociale des textes (et non créés ad hoc pour l'enseignement) qui en manifestent concrètement la variabilité. Cette exposition à la variation des textes actualisant un même genre discursif devrait aussi aller croissant à mesure des acquisitions dans la languecible. 


\section{L'approche par genres de discours de l'enseignement de la langue de scolarisation}

La langue de scolarisation a pour fonction essentielle de faire accéder les élèves aux connaissances : elle est le vecteur de tous les savoirs enseignés. L'acquisition des connaissances en contexte scolaire, quels que soient les contenus disciplinaires (mathématiques, géographie, biologie...) passe par le langage, même si elle ne se réduit pas à des acquisitions langagières. Les difficultés d'apprentissage rencontrées par les élèves - en particulier ceux issus de milieux défavorisés ou de la migration - sont reconductibles tout aussi bien aux décalages entre leurs savoirs spontanés et les savoirs savants qu'à leur expérience de la langue de scolarisation. Pour tous les élèves cependant, l'entrée dans l'École constitue bien un saut discursif, que peut masquer l'usage d'une langue réputée connue (pour ce qui est des enfants locuteurs natifs).

\subsection{Le « saut discursif»}

La construction des connaissances et des compétences dépend largement de la maîtrise des discours scientifiques et techniques qui sont produits dans la langue de scolarisation. Les formes de la communication scientifique sont déterminantes pour la construction et la transmission des connaissances, car, le langage n'est pas uniquement l'instrument privilégié de la communication sociale : il a toujours été utilisé pour fixer et stocker des informations et des connaissances. Ces formes verbales données aux connaissances en ont assuré la transmission dans le temps et elles ont permis les controverses scientifiques, qui sont fondamentales pour l'évolution des savoirs. Si certaines connaissances peuvent être communiquées dans des systèmes sémiotiques non verbaux (symboles, formules, cartes, schémas...), elles doivent nécessairement être verbalisées pour la discussion, le commentaire ou l'enseignement. De plus, des disciplines comme les sciences sociales/humaines n'utilisent pas le langage simplement pour représenter des connaissances déjà établies en dehors des textes, lesquels se borneraient à en rendent compte : certaines connaissances n'ont d'existence que verbale. Ainsi n'y a-t-il pas de connaissance historique en dehors des textes des historiens, même si celle-ci s'élabore à partir de sources, de données et d'indices de toute nature.

On peut avancer que

«[...] la maîtrise des connaissances ne se réduit aucunement à celle du langage qui permet de les exprimer [...] Le langage des apprenants utilisé dans les matières scientifiques, artistiques et techniques (dont, partiellement, les enseignements de la/des langue/s de scolarisation) est à considérer par les enseignants de ces matières comme un ensemble d'indices de surface permettant indirectement d'observer leurs acquis cognitifs. Mais, inversement en quelque sorte, si les apprenants acquièrent les "bons moyens" linguistiques de communiquer relativement aux connaissances, alors cette maîtrise est peut-être en mesure d'influencer positivement leurs acquisitions et de les amener aux postures et démarches scientifiques recherchées. » (Beacco, J.-C., Coste, D., van de Ven, P.-H. \& Vollmer, H., 2010 : 11). 
De plus, les champs scientifiques et techniques se définissent par des méthodologies partagées d'élaboration de la connaissance mais aussi par des conventions relatives à la communication (verbale) dans ces communautés qui concernent la forme des textes produits : thèse, communication à un colloque, rapport d'expérimentation, publication d'un chantier de fouilles... Apprendre une science signifie donc devenir capable de comprendre et de produire des textes conformes à la culture du domaine. Du point de vue des enseignements scolaires du cycle primaire, cela veut dire, en gros, faire passer les élèves des discours en je-ici-maintenant, celui de la communication ordinaire, à des genres de discours objectivés, généralisables et fondés/argumentés qui au moins préfigurent des genres de discours véritablement propres aux communautés scientifiques

\subsection{Une décentration discursive par continuum}

Alors que pour l'acquisition d'une compétence discursive en langue étrangère, il convient d'exposer les apprenants à des exemples authentiques de textes à produire, des stratégies éducatives spécifiques sont nécessaires pour faire passer les élèves des discours ordinaires centrés sur le «je », sur l'expérience immédiate et sur l'environnement proche à des discours distanciés et objectivés. Car ce parcours est à la fois cognitif et linguistique et non exclusivement linguistique.

De telles démarches ${ }^{(7)}$ ont été mises en œuvre dans les activités d'éveil scientifique, telles que l'élevage d'animaux, l'entretien d'un jardin scolaire, les observations en forêt (par ex. recherche de champignons)... qui donnent lieu à des échanges entre pairs, à une exploration autonome du milieu et à des formes de mobilisation de la fonction symbolique. Du point de vue de la communication verbale, ces activités suscitent d'abord des interactions, où sont exprimées et échangées des opinions (— «Y a des noisettes »— «Non, c'est des glands »), des suppositions, des questions... La communication entre les enfants permet de passer des évidences à la confrontation des opinions et à leur justification sous la conduite du maître. Les dessins, schémas croquis, maquettes jouent un grand rôle pour le passage à l'abstraction. Ces activités peuvent se poursuivre par la mise en place d'expérimentations simples, qui vont donner lieu à un compte-rendu. Et la distinction qui va s'opérer entre compte-rendu et récit/narration est fondamentale pour les processus d'objectivation. Car, des textes de cette nature comportent des éléments comme : préciser (des circonstances), décrire (l'aspect, le processus), établir des relations (moyen, but), mesurer, formuler des doutes/des réserves, généraliser, comparer décrire, classer... Les éléments constitutifs de ce «genre » sont essentiellement des opérations cognitives, c'està-dire les représentations (au sens de mise en scène/en mots) discursives des processus cognitifs activés pour l'élaboration ou l'exposition du savoir, comme : calculer, classifier, comparer, décrire/représenter, déduire... Pour chacune, il est possible d'identifier les ressources linguistiques nécessaires à leurs réalisations.

(7) L'essentiel de ce développement provient de Collectif (1983) : « Eveil scientifique et modes de communication ", Recherches pédagogiques 117, Institut National de la Recherche Pédagogique. 
On voit donc que la démarche pédagogique des activités d'éveil scientifique permet de passer d'interactions spontanées à des échanges d'opinion, puis à des discussions argumentées, à des schématisations, à des réponses organisées par un protocole expérimental et enfin au compte-rendu d'une expérimentation. Or, celui-ci qui n'est pas un genre de discours figurant dans le répertoire ordinaire individuel des élèves et ce n'est pas non plus un genre scientifique : c'est un genre scolaire, propre à cette communauté de communication (comme la rédaction ou la dissertation) relativement indifférencié (c'est-à-dire à régulations génériques faibles). On peut le considérer comme un pré-genre de discours scientifique. L'accès aux connaissances transite par l'accès à ce nouveau genre de discours, auquel on est conduit par médiation à travers les genres discursifs ordinaires (les conversations).

Un tel continuum est de nature à permettre de faire passer les élèves des formes ordinaires de la communication à certaines de ses formes scientifiques. La « question de la langue » dans l'enseignement des matières scolaires n'est pas à renvoyer à une sorte de responsabilité collective des enseignants, laquelle tend de ce fait à se diluer. Il ne s'agit pas seulement de transformer tout enseignant de discipline en enseignant de français, chargé de veiller au bon emploi générique de la langue (morphologie, vocabulaire, orthographe...). Cette responsabilité incombe certes à chacun, mais les enseignants de disciplines ont un rôle spécifique parce qu'il existe spécificité de communication scientifique et que celle-ci ne réside pas uniquement dans la terminologie utilisée.

\subsection{Les dimensions linguistiques des programmes scientifiques}

Il n'est pas possible de décrire ici dans le détail, les étapes conduisant à la réalisation d'un « programme linguistique » pour une matière scolaire. On se bornera à en rappeler les éléments constitutifs. Ils devraient comporter :

— un inventaire des compétences disciplinaires visées ; par exemple, pour 1'histoire : formuler les bonnes questions à propos des documents disponibles ; analyser les sources d'information disponibles et faire la distinction entre sources primaires et secondaires...;

- un inventaire des composantes de la connaissance visées : catégories et connaissances générales (par ex. toujours pour l'histoire : longue durée, ?civilisation/culture, multicausalité..), spécifiques ou locales, proches et lointaines dans l'espace et dans le temps (naissance de l'économie capitaliste, mémoire collective, ostracisme (Grèce), Risorgimento (Italie...);

- un recensement des formes de la communication (sous forme de genres de discours) effectivement utilisées dans l'enseignement/apprentissage et de leurs rôles respectifs ;

- pour chaque genre de discours, on doit alors développer des descripteurs en termes de capacités langagières requises pour gérer (en réception ou production) le genre donné ; par exemple, pour le genre « exposé » : interpréter des données quantitatives ou « développer un exposé de manière claire et méthodique, en en soulignant les points significatifs » (descripteur B2 du Cadre européen de référence pour les langues, p. 50). 
A partir de ces spécifications, on peut redescendre aux éléments constitutifs des genres de discours, comme «définir». Définir, en histoire peut se réaliser par une série d'exemples, par une/des comparaisons, par l'étymologie.. ce qui renvoie à la maîtrise de formes linguistiques comme : par exemple, à titre d'exemple, ces exemples montrent que..., d'après ces exemples...

Pour chaque matière scolaire, il importe donc de décrire clairement la forme des textes oraux et écrits attendus des apprenants, en tant qu'ils relèvent d'un genre de discours lui-même bien identifié, de manière à éviter les effets de «curriculum caché », et de bien distinguer les textes servant à l'appropriation des connaissances de ceux utilisés pour l'évaluation des acquisitions.

L'emploi de la catégorie genre de discours est déterminant dans une didactique visant à apprendre à produire des textes pour faire acquérir des formes linguistiques peu ou non connues. Le choix des genres à privilégier pour les apprentissages relève de critères multiples dont la régularité et la prévisibilité des textes actualisant un genre, leur proximité d'avec certains genres faisant déjà partie du répertoire individuel ou la pertinence des contenus abordés en fonction de la nature de la motivation des apprenants. Pour les langues étrangères, on a généralement fait le choix de l'authenticité, c'est-à-dire de l'exposition à des échantillons de textes extraits tels quels de la circulation sociale des textes et constituant des modèles pour la production ou pour mettre en place des stratégies stables de reconnaissance. Pour ce qui est de l'accès aux connaissances en langue de scolarisation, on peut choisir de faire faire aux apprenants l'expérience de genres de textes (compte-rendu, dissertation...) n'ayant pas d'utilisation hors de la communauté de communication que constitue l'École. Ces formes jouent un rôle d'intermédiaire entre les répertoires premiers et les genres scientifiques, où sont mises en jeu des compétences cognitives et linguistiques. Privilégier une stratégie facilitatrice (en utilisant des genres « mixtes » ou artificiels) ou une stratégie de l'authenticité générique est avant tout affaire de contexte et d'apprenants. Il n'en reste pas moins que, bien que les genres de discours soient une catégorie métalinguistique ordinaire, les responsables de programme et, à leur suite les enseignants, demeurent peu enclins à les utiliser et continuent à privilégier la tradition des didactiques centrées sur la phrase et non sur le texte. 


\section{Bibliographie}

BAKHTINE, M. (1929) : Marxisme et philosophie du langage, cité par Todorov, T. (1981) : Mikhaïl Bakhtine Le principe dialogique suivi de Ecrits du Cercle de Bakhtine, Paris, Seuil.

BEACCO, J.-C. (1991) : «Types ou genres? Catégorisation des textes et didactique de la compréhension et de la production écrites », Etudes de linguistique appliquée 83, p. 19-28.

— dir. (1999) : L'astronomie dans les médias. Analyses linguistiques de discours de vulgarisation, Paris, Presses de la Sorbonne nouvelle.

- (2004) : «Trois perspectives linguistiques sur la notion de genre discursif », Langages 153, p. 109-119, Paris, Larousse.

- (2009) : Eléments pour une description des compétences linguistiques en langue de scolarisation nécessaires à l'enseignement/apprentissage de l'histoire dans le premier cycle de l'enseignement secondaire : une démarche et des points de référence, dans Langues de l'éducation, langues pour l'éducation. Plate-forme de ressources et de références pour l'éducation plurilingue et interculturelle, Conseil de l'Europe http ://www.coe.int/t/dg4/linguistic/Source/Source2010_ForumGeneva/1_LIS-History2010_fr.pdf.

Beacco, J.-C., LePAGe, S, Porquier, R \& RiBA, P. (2008) : Niveau A2 pour le français, Paris, Didier.

BeAcco, J.-C., Coste, D., VAN DE Ven, P.-H. \& Vollmer, H. (2010) : Langues et matières scolaires - Dimensions linguistiques de la construction des connaissances dans les curriculums, Strasbourg, Conseil de l'Europe.

Burns, A. (2013) : "Genre and genre based teaching », Byram M. \& Hu A. (eds) : Routledge Encyclopedia of Language Teaching and Learning, Oxon, Routledge, p. 269-274.

COLlECTIF (1983) : Eveil scientifique et modes de communication, Recherches pédagogiques 117 , Paris, INRP .

CONSEIL DE L'EUROPE (2001) : Cadre européen de référence pour les langues. Apprendre, enseigner, évaluer, Strasbourg, Conseil de l'Europe et Paris, Didier.

Coste, D, De Pietro, J.-F. \& Moore, D. (2012) : « Hymes et le palimpseste de la compétence de communication », Langage et société 139, p.103-123.

Coste, D., MOORE, D., ZARATE, G. (2009 vers. révisée) : Compétence plurilingue et pluriculturelle, Strasbourg, Conseil de l'Europe. http ://www.coe.int/t/dg4/Linguistic/Source/SourcePublications/CompetencePlurilingue09web_FR.pdf.

Charaudeau, P. (2002) : «Genre de discours » dans Charaudeau, P et Maingueneau, D. (dir) : Dictionnaire d'analyse du discours, Paris, Seuil, p. 277280.

HyMES, D. (1972, traduction française 1980) : « Modèles pour l'interaction du langage et de la vie sociale », Etudes de linguistique appliquée $\mathrm{n}^{\circ} 37$, p. 125 153.

Maingueneau, D. (2002) : «Analysis of an academic genre », Discourse Studies vol. 4-3, p. 319-341. 